prognosis. The rarity of this tumor makes the risk of its development undetermined.

\section{EPV113/\#323 PROGNOSTIC FACTORS AND ONCOLOGIC OUTCOMES FOR PATIENTS TREATED WITH ADJUVANT CHEMOTHERAPY AND VAGINAL VAULT BRACHYTHERAPY FOR STAGE I ENDOMETRIAL SEROUS CARCINOMAS}

${ }^{1} \mathrm{~V}$ Dorion*, ${ }^{2} \mathrm{~V}$ Samouëlian, ${ }^{1} \mathrm{M}-\mathrm{C}$ Beauchemin, ${ }^{2} \mathrm{~B}$ Cormier, ${ }^{2} \mathrm{E}$ Tremblay, ${ }^{1} \mathrm{M}$ Barkati. ${ }^{1}$ Centre hospitalier de I'Université de Montréal, Radiation Oncology, Montreal, Canada; ${ }^{2}$ Gynecologic Oncology Service, CHUM, Université de Montréal, Department of Obstetrics and Gynecology, Montreal, Canada

\subsection{6/ijgc-2021-IGCS. 183}

Objectives Endometrial serous carcinomas (ESC) hold a poor prognosis, even at early stages. This study evaluates the outcomes and prognostic factors for stage I (FIGO 2018) ESC treated with adjuvant chemotherapy and vaginal vault brachytherapy (VBT).

Methods Patients were selected through a database of patients treated with hysterectomy for stage I ESC between 2007 and 2019 at the Centre Hospitalier de l'Université de Montréal. The intended adjuvant treatment had to be 6 cycles of Carboblatin and Paclitaxel and VBT. Time to events were analyzed by Kaplan-Meier. Cox regression analysis was performed to identify prognostic factors.

Results A total of 76 patients with stage IA $(\mathrm{N}=64)$ and IB $(\mathrm{N}=12)$ ESC were included in this study. Median age at diagnostic was 67. Median follow up was 60 months. 5-year overall survival (OS) and progression-free survival (PFS) were 83\% and $79.5 \%$. Nine patients relapsed, 3 with local recurrence, 3 with regional recurrence and the other 3 with distant recurrence. Amongst the known prognostic factors included in univariate analysis, positive peritoneal washing and advanced age were significant prognostic factors for OS $(p<0.0001$ and $\mathrm{p}=0.013$, respectively). Age, isthmus invasion, deep myometrial invasion and positive peritoneal washings were significant prognostic factors for PFS $(p=0.049, p=0.024, p=0.022$ and $\mathrm{p}<0.0001$, respectively).

Conclusions In stage I ESC, adjuvant chemotherapy and VBT was associated with good oncologic outcomes. Advanced age and positive peritoneal washings were significant prognostic factors for OS. Further studies are needed to assess whether a subgroup of patients would benefit from treatment intensification or de-escalation.

\section{EPV114/\#325 EXPRESSION OF AQUAPORINS IN HUMAN ENDOMETRIAL CANCER: IDENTIFICATION AND REGULATION BY OVARIAN HORMONES IN CARCINOGENESIS OF ENDOMETRIAL CANCER}

${ }^{1} S$ Khan*, ${ }^{1} \mathrm{~A}$ Yool, ${ }^{2} \mathrm{C}$ Ricciardelli. 'Adelaide Medical School, Univeristy of Adelaide, Physiology, Australia/Adelaide, Australia; ${ }^{2}$ Adelaide Medical School, Univeristy of Adelaide, Discipline of Obstetrics and Gynaecology, Robinson Research Institute, Australia/Adelaide, Australia

\subsection{6/ijgc-2021-IGCS. 184}

Objectives Aaquaporins contribute to pathogenesis of Endometrial cancer. Our study presents the first screen of grade I and grade III endometrial cancer cell lines for all 13 AQP classes in response to physiological doses of estrogen and progesterone.

Methods Ishikawa (IKC, grade I) and MFE-280 (grade III) were assessed with estrogen and progesterone at relevant doses, at multiple time points for cell proliferation, motility (3D migration and invasion assays), and cytoskeletal organisation. Patterns of AQP expression were compared in IKC and MFE-280 by quantitative (q) PCR and western blot (WB).

Results Cell numbers, 3D migration and invasiveness were increased in IKC by estrogen and decreased by progesterone in a dose- and time-dependent manner. Estrogen induced formation of lamellipodia in IKC. The EC50 and IC50 values for estrogen and progesterone were $1 \mathrm{nM}$ and $100 \mathrm{nM}$ respectively. Transcript levels of AQPs $0,-2,-3,-4,-5,-8$ were significantly decreased by estrogen and progesterone in IKC, whereas AQP11 and AQP12 were increased. In contrast, in MFE-280 cells, estrogen and progesterone caused an increase in transcript levels for AQPs 3,-4,-7, -8, whereas expression of AQPs 0 , and -11 were decreased. Protein expression of AQP-1 and -4 was confirmed by WB.

Conclusions These findings indicate the potential role of aquaporins in progression and invasion of endometrial cancer, and highlight the previously unstudied AQPs 11 and 12 as targets of potential interest. Outcomes here provide a foundation for further exploration of aquaporin inhibitors in decreasing the progression of EC, and insights into new therapeutic strategies

\section{EPV115/\#333 INTERIM ANALYSIS OF 10-YEAR DATA REGARDING PRESENTATION AND MANAGEMENT OF UTERINE CARCINOSARCOMA (UCS) CASES ACROSS THE THAMES VALLEY CANCER ALLIANCE NETWORK}

${ }^{1}$ A Gkorila* ${ }^{2} S$ Smyth ${ }^{3} \mathrm{P}$ Tupper ${ }^{4} \mathrm{~A}$ Mcculloch ${ }^{1} \mathrm{~N}$ Sadeghi, ${ }^{1} \mathrm{~V}$ Le Thanh, ${ }^{1} \mathrm{~A}$ Sattar ${ }^{1} \mathrm{~S}$ Spencer, ${ }^{1} \mathrm{~K}$ Zarrindej, ${ }^{2} \mathrm{M}$ Abdalla, ${ }^{2} \mathrm{~S}$ Addley, ${ }^{1} \mathrm{~J}$ Rencher, ${ }^{1} \mathrm{G}$ Sharma, ${ }^{1} \mathrm{~A}$ Kashif, ${ }^{5} \mathrm{~S}$ Siddiki, ${ }^{2} \mathrm{M}$ Alazzam, ${ }^{2} \mathrm{H}$ Soleymani Majd. ${ }^{1} \mathrm{Oxford}$ University Hospitals NHS Foundation Trust, Obstetrics and Gynaecology, Oxford, UK; ${ }^{2}$ Oxford University Hospitals NHS Foundation Trust, Gynaecological Oncology, Oxford, UK; ${ }^{3}$ Buckinghamshire Healthcare NHS Trust, Obstetrics and Gynaecology, Aylesbury, UK; ${ }^{4}$ Royal Berkshire Hospital NHS Foundation Trust, Obstetrics and Gynaecology, Reading, UK; ${ }^{5}$ Great Western Hospitals NHS Foundation Trust, Obstetrics and Gynaecology, Swindon, UK

\subsection{6/ijgc-2021-IGCS. 185}

Objectives UCS comprises $<5 \%$ of uterine malignancies, accounting for $>15 \%$ associated mortality. With no established guidelines, we present our experience to determine clinical characteristics, treatment modalities and histology outcomes of UCS.

Methods We conducted a multicentre retrospective cohort study, including all surgically managed UCS cases between March 2010 and January 2020. Data was collected on patients' demographics, medical history, pre-operative and final histology and FIGO staging, peri-operative and post-operative findings.

Results 82 (9.7\%) UCS cases were identified from a total of 847 surgically managed uterine cancers, with 51 diagnosed with UCS. 3 cases were down and 12 up-staged following surgery. 15 cases of MRI lymphadenopathy led to a PPV of $40 \%$. Positive lymph nodes and omentum were identified in $15.8 \%$ and $11.3 \%$ of cases respectively, with half of lymph node metastases diagnosed following systematic dissection (the majority of which were LVSI positive). There were no operative complication themes. 
Abstract EPV115/\#333 Table 1 Pre-operative characteristics and ivestigations of patients with UCS

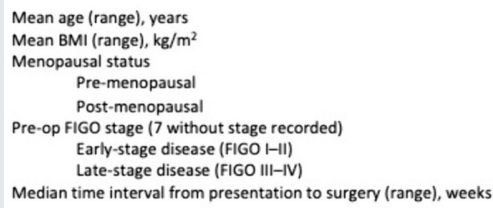

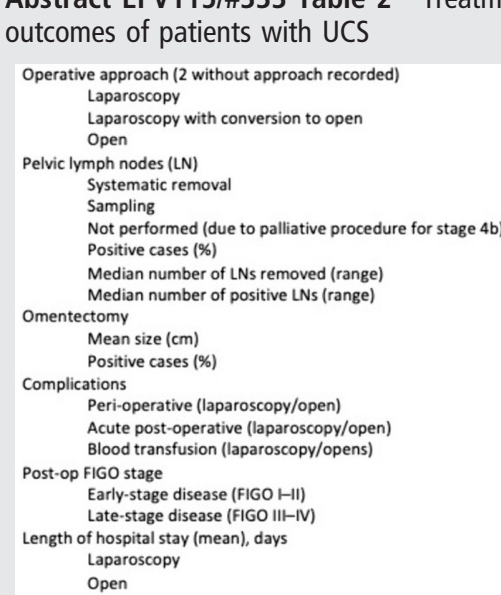
outcomes of patients with UCS

Abstract EPV115/\#333 Table 2 Treatment modalities and

Conclusions UCS presented with almost double the incidence rate previously described. The overall accuracy of pre-operative staging was $81 \%$. Our analysis showed that $86 \%$ of cases were managed laparoscopically, with more favourable peri-operative and post-operative profiles. Despite the lack of management guidelines, we stress the importance of urgent surgical treatment for UCS, in the form of total hysterectomy, bilateral salpingo-oophorectomy, systematic bilateral pelvic lymph node dissection and omentectomy. Data on adjuvant treatment, recurrence and survival is currently under analysis.

\section{EPV116/\#34 HIGH GRADE ENDOMETRIAL CARCINOMA LIMITED TO THE ENDOMETRIUM OR A POLYP. IS ADJUVANT TREATMENT NECESSARY?}

L Dallaire Nantel*, MC Renaud, A Sebastianelli, J Grégoire, M Plante. Hotel Dieu de Quebec, Gynecology Oncology, Quebec, Canada

10.1136/ijgc-2021-IGCS. 186

Objectives High grade endometrial carcinoma limited to the endometrium or a polyp is a rare clinical entity. Currently there is no consensus on standard treatment. Thus, the goal of this study was to evaluate the clinical outcomes of patients with type II endometrial carcinoma without myometrial infiltration or limited to a polyp.

Methods We retrospectively identified type II endometrial carcinoma with spread limited to the endometrium or a polyp from April 2013 to November 2017. Medical records were reviewed for the following information: age at diagnosis, characteristics of patients, type of surgery, histology, stage according to FIGO 2009 classification, adjuvant treatments, and site of recurrence. Descriptive statistics and the KaplanMeier estimate were used for analysis.

Results Twenty-six patients with a type II stage IA adenocarcinoma were included. All were surgically staged with total hysterectomy, salpingo-oophorectomy and lymph nodes assessment. The median age at diagnosis was 69 years. All patients had either disease limited to the endometrium (61.5\%) or a polyp (38.5\%). Only four patients had lymphovascular space invasion (16.5\%). Median follow up was 44 months (2-75 months). Most patients did not receive adjuvant treatment after surgery (73\%). Three patients (11.5\%) experienced recurrences 15, 21 and 55 months after surgery. Following systemic treatment all are alive and free of disease. The 3-year progression free survival and overall survival were $91 \%$ and $100 \%$ respectively.

Conclusions Based on our data, expectant management with surveillance alone following surgery appears to be safe for patients with high-grade endometrial carcinoma limited to a polyp or the endometrium without myometrial invasion.

\section{EPV117/\#36 PD-L1 IS A TUMOR SUPPRESSOR IN AGGRESSIVE ENDOMETRIAL CANCER CELLS AND ITS EXPRESSION IS REGULATED BY MIR-216A AND LNCRNA MEG3}

P Dong*. Hokkaido University, Department of Obstetrics and Gynecology, Sapporo, Japan

\subsection{6/ijgc-2021-IGCS.187}

Objectives Poorly-differentiated endometrioid adenocarcinoma and serous adenocarcinoma represent an aggressive subtype of endometrial cancer (EC). Programmed death-ligand-1 (PD-L1) was known to exhibit a tumor cell-intrinsic function in mediating immune-independent tumor progression. However, the functional relevance of tumor cell-intrinsic PD-L1 expression in aggressive EC cells and the mechanisms regulating its expression remain unknown.

Methods PD-L1 expression in 65 EC tissues and 18 normal endometrium samples was analyzed using immunohistochemical staining.

Results Positive PD-L1 expression was identified in $84 \%$ of benign cases but only in $12 \%$ of the EC samples, and the staining levels of PD-L1 in EC tissues were significantly lower than those in the normal tissues. Higher PD-L1 expression predicts favorable survival in EC. Ectopic expression of PD-L1 in aggressive EC cells results in decreased cell proliferation and the loss of mesenchymal phenotypes. Mechanistically, PDL1 exerts the anti-tumor effects by downregulating MCL-1 expression. We found that PD-L1 levels in aggressive EC cells are regulated by miR-216a, which directly targets PD-L1. We further identified a mechanism whereby the long non-coding RNA MEG3 represses the expression of miR-216a, thereby leading to increased PD-L1 expression and significant inhibition of cell migration and invasion.

Conclusions These results revealed an unappreciated tumor cell-intrinsic role for PD-L1 as a tumor suppressor in aggressive EC cells, and identify MEG3 and miR-216a as upstream regulators of PD-L1. 